\title{
Ant colony optimization approach based on precedence constraint matrix for flexible process planning
}

\author{
Fengli Huang*, Haiyan Wang, Jinmei Gu and Qun Sun \\ School of Mechanical and Electrical Engineering, Jiaxing University, Jiaxing 314001, China \\ *Corresponding Author: hfl@mail.zjxu.edu.cn
}

\section{INTRODUCTION}

In contemporary integrated manufacturing system, the CAPP (Computer Aided Process Planning) is for the PPS (Production Planning and Scheduling); meanwhile, it also needs the support of PPS system. The concurrent integration of CAPP and PPS, which formed the IPPS (Integrated Process Planning and Scheduling) system, is of great importance to improve equipment utilization, eliminate manufacturing resource conflicts, and reduce workpiece circulation time (Seker et al., 2013; Li et al., 2015; Zhang et al., 2015). However, the traditional CAPP is static, which may lead to the failure of the process plan (Usher et al., 1996). The process decisions are made under the assumption that the manufacturing resources are free at any time, without considering the plant dynamic events or equipment bottlenecks and other issues. Therefore, the process planning should be based on the specific manufacturing resources and generate a flexible process planning program, which can be dynamically integrated with the PPS system.

Flexible process planning implies the ability of a system to follow change requirements and thereby provide alternative ways of performing manufacturing operations on a part. In order to adapt to the dynamic environment of manufacturing resources and integrate with PPS system, process planning system should generate a large number of flexible process plans for each part, which is optimized and selected according to the manufacturing demands (Li et al., 2008). The flexible process planning, which is affected by process precedence constraints, involves many aspects, as the determination of sequence of operations, selection of machine tools, selection of cutting tools, selection of fixtures and so on, and it is also affected by process precedence constraints. As a result, the optimization and selection of flexible process planning are an NP-Complete problem (Petrović et al., 2015). The conventional non- heuristic methods cannot provide an optimal solution for this combinatorial problem. Thus, many researchers employed computational intelligence-based algorithms, such as genetic algorithm (GA), simulated annealing (SA), tabu search (TS), particle swarm optimization (PSO), ant colony optimization (ACO), and hybrid algorithms, to solve this problem.

Yiphoi et al. (1996) discussed various aspects of parallel machining that influence the generation of process plans and proposed a process planner by a genetic algorithm for sequencing operations. Qiao et al. (2010) proposed four types of process planning rules including precedence rules, clustering rules, adjacent order rules, and optimization rules in the fitness calculations for alternative operation sequences. They investigated the impact of various rules on the result of operation sequencing and that of genetic algorithms parameters on the solution efficiency, as well as the influence of manufacturing resource environment on the process planning. Huang et al. (2011) developed a solution framework for complex parts based on genetic algorithms approach combined with operation precedence graph (OPG). With modified initial population, crossover operator and mutation operator, this approach is able to optimize the process planning by simultaneously considering the assignment of machining resources, determining sequencing operation and setup plans.

Brown et al. (1997) proposed an alternative approach to accomplish the process planning of a part by decomposing the part into several features and determining the process method according to the feature description, and then 
applying a stochastic search technique according to simulated annealing to obtain a process plan. Considering the processing costs, G. H. Ma et al. (2000) proposed a process planning model for generating a feasible plan for a given part based on variable machining resources, and an algorithm based on simulated annealing was developed to search for the optimal solution. Musharavati et al. (2012) applied three simulated annealing algorithms that exploit auxiliary knowledge in different ways to handle a manufacturing process planning problem for reconfigurable manufacturing. The computational analysis shows that the simulated annealing algorithms that are supported by auxiliary knowledge can obtain a better optimal solution than that without auxiliary knowledge support. Li et al. (2004) utilized the tabu search based approach to solve the process planning problem under the machining and cutting tools changes.

Wang et al. (2011) took advantage of particle swarm optimization (PSO) to solve the process planning problem. In this approach, a solution representation scheme is introduced for the application of PSO, and two kinds of local search algorithms are incorporated and interweaved with PSO evolution to find the best solution of operation-method selection and sequencing. Li et al. (2013) proposed a modified PSO algorithm, in which the efficient encoding, updating, and random search methods are improved, to optimize the process planning problem, and instance analysis shows that modified PSO algorithm can generate satisfactory solutions and outperform the genetic algorithm and simulated annealing algorithm. Milica et al. (2015) developed a new algorithm based on the utilization of PSO algorithm incorporated with chaos theory to optimize the flexible process plans, and experimental studies show that the new algorithm has a better performance than GA, SA, hybrid GA-SA, and generic PSO based approach. Considering the variety of alternative machines, alternative tools, as well as tool access direction (TAD), Zoran et al. (2016) established a multi-objective optimization model for flexible process planning and developed a modified PSO to solve the nondeterministic combinatorial optimization problem.

Krishna et al. (2006) applied the ant colony algorithm as heuristic search technique to optimize the operations sequence by considering the various feasibility constraints of processing. In view of the cost for machining process, Liu et al. (2013) applied an ant colony optimization algorithm to solve the process planning problem, which is involved with the selection of the available machining resources, the sequence of machining operations, and the manufacturing constraints. Wang et al. (2015) represented the process planning problem as a directed graph that consists of nodes, directed/undirected arcs, and OR relations and utilized a two-stage ant colony optimization approach to solve the process planning problem based on the graph.

In flexible process planning, there are complex constraint precedence relationships in the processing operations. The key point of algorithm designing to solve the operational sequencing problem is to deal with the constraint precedence relationship. Researchers have used many methods to describe the problem of the processing operation constraint, such as Petri-net (Kiritsis et al., 1996), operation precedence graph (Wang et al., 2015), process plan network (Sormaz et al., 2003), and AND/OR graphs (Li et al., 2008). However, these methods are unable to solve the problem of dynamic updating of the constraint relationship among the processing operations. In this paper, the precedence constraint relationship was described by precedence constraint matrix, and the dynamic updating of the operation constraints was represented by the updating of precedence constraint matrix. The dynamic updating algorithm of operation constraints was incorporated with ant colony algorithm, which was used to find the optimal solution to flexible process planning problem in limited search space.

\section{FLEXIBLE PROCESS PLANNING PROBLEM AND PREFERENCE CONSTRAINT MATRIX}

In part processing, manufacturing features can generally be used to describe the geometric structure of the parts that need to remove materials, the corresponding size tolerance, position tolerance, surface roughness, and other technical requirements.

The flexible process planning can quickly get the optimal process route by resetting the alternative manufacturing resources when the response manufacturing environment changed. The process flexibility of parts can be divided into three types: machining sequence flexibility, operation flexibility, and manufacturing resources flexibility (Li et 
al., 2008; Wen et al., 2012). Machining sequence flexibility means that some processing operations of parts can interchange, mainly because the processing parts have multiple manufacturing characteristics; each manufacturing characteristic may have $n_{o p}\left(n_{o p} \geq 1\right)$ machining operations. Since precedence constraints exist in partially machining operations and do not exist in other machining operations, the same part has a variety of processing order (Lv et al., 2013). The flexibility of machining operation means that the same manufacturing feature can be finished by choosing the different machining operation, resulting in various processing schemes for each manufacturing feature; each machining scheme contains different machining operations. The flexibility of manufacturing resources suggests that the machining operation of parts can be done by making use of different manufacturing equipment.

$O P$ is machining operation, and $A O$ is the set composed by machine tool $(M)$ and tool $(T)$ and tool access direction $(T A D)$. Thus, it can be seen that the sequencing orders of machining operation are the more important work of process planning for a certain part; the process route can be expressed by the following formula:

$$
P P=\left\{O P_{1}, O P_{2}, \cdots, O P_{i}\right\}
$$

$O P_{i}$ is the $i$-th machining operation of parts and can be expressed by the following formula:

$$
O P_{i}=\left\{A O_{i 1}, A O_{i 2}, \cdots, A O_{i j}, \cdots, A O_{i n}\right\}
$$

$A O_{i j}$ is corresponding to the $j$-th alternative operation of the $i$-th machining operation of parts and can be expressed by the following formula:

$$
A O_{i j}=\left\{M_{i j}, T_{i j}, T A D_{i j}\right\}
$$

$M_{i j}, T_{i j}$ and $T A D_{i j}$ represent, respectively, machine tool, tool, and tool feed direction of the alternative machining operation $A O_{i j}$. All the above alternative machining operations can be consisting of machining operations set, which is recorded as 0 . Table 1 states any $O_{i}$ of the alternative machining operation set.

Table 1. Class definition of an alternative machining operation.

\begin{tabular}{ll}
\hline Variable $i$ & Description \\
\hline Alternative Operation_id & The alternative machining operation ID \\
Machine_id & The machine ID to execute the alternative machining operation \\
Machine_list & The candidate machine list for executing the alternative machining operation \\
Tool_id & The tool ID to execute the alternative machining operation \\
Tool_list & The candidate tool list for executing the alternative machining operation \\
TAD_id & The TAD ID to apply the alternative machining operation \\
TAD_list & The candidate TAD list for executing the alternative machining operation \\
\hline
\end{tabular}

There are precedence constraints in the machining operations because of the geometrical shape of manufacture feature and the constraint of manufacture process. This will be mainly embodied in the intersection occlusion of the manufacturing feature in the process of machining and the technological requirements. The usual processing precedence constraints mainly have the following situation:

(1) When the manufacture characteristics are machined on the same surface, they should strictly abide by the order of the roughing, semi-finishing, and finish machining.

(2) For the parts with surface and hole, if the plane is the datum of the hole, then abide by the surface after the hole. 
(3) The reference surface should be machined prior to the machining feature associated with it.

(4) Main surface should be machined first, and then the minor surface is machined.

(5) If the two features intersect, along the feed direction, the obscured machining features should be machined later.

The problem of processing priority was expressed by using the operation precedence graph in more literatures (Huang et al., 2011; Lee et al., 2004); after processing priorities were expressed by making use of operator precedence graph, the operator precedence graph is used to ensure the feasibility of the processing sequence. In the actual machining process, operator precedence graph cannot express complicated processing priority constraint relation, especially the multi-level nested preference constraint relationship. In the choice process of machining operations, there will be dynamic change situation. The precedence constraint matrix can conveniently express preference constraint relationship in the alternative machining operations (Huang et al., 2016). Suppose there is $N$ alternative operations in set 0 ; Nth-order matrix is defined to store the preference constraint relationship in alternative operations, as shown in formula (4):

$$
\mathrm{C}=\left[\begin{array}{cccc}
C_{11} & C_{12} & \cdots & C_{1 N} \\
C_{21} & C_{22} & \cdots & C_{2 N} \\
\vdots & \vdots & & \vdots \\
C_{N 1} & C_{N 2} & \cdots & C_{N N}
\end{array}\right]
$$

where $O_{i j}$ represents the preference constraint relationship between $O_{i}$ and $O_{j}, i, j=1,2, \cdots, N$, the value is defined by the following rules: when the $i$-th alternative operation is prior to the $j$-th alternative operation, then $C_{i j}=1$, $C_{i j}=0$. When $O_{i}$ and $O_{j}$ belong to the same operation $O P_{k}$, then $C_{i j}=C_{i j}=-1$, under other cases, $C_{i j}=C_{i j}=0$. Obviously, there is $C_{i j}=\{-1,0,1\}$.

Precedence constraints determined by the processing technology are in the layer of machining operation $O P_{i}$, then all alternative machining operations of $O P_{i}$ have the same precedence constraints. It is related to the tool access direction whether intersection features possess obscured precedence constraints, As a consequence, the precedence constraints are in the layer of alternative machining operations $A O_{i j}$; then the alternative machining operations in the same machining operation $O P_{i}$ have the different precedence constraints. The different machines and tools in the alternative operation set $A O_{i j}$ of machining operation $O P_{i}$ do not influence the preference constraint relations; therefore, the alternative machining operation $O P_{i}$ will be redefined that it is only related with the direction of $T A D_{i j}$ and is as follows:

$$
A O_{i j}=\left\{M, T, T A D_{i j}\right\}
$$

Suppose there is processing part as shown in figure 1 (Wang et al., 2015); part blank is the square envelope body, and processing operations of features are shown in table 2 :

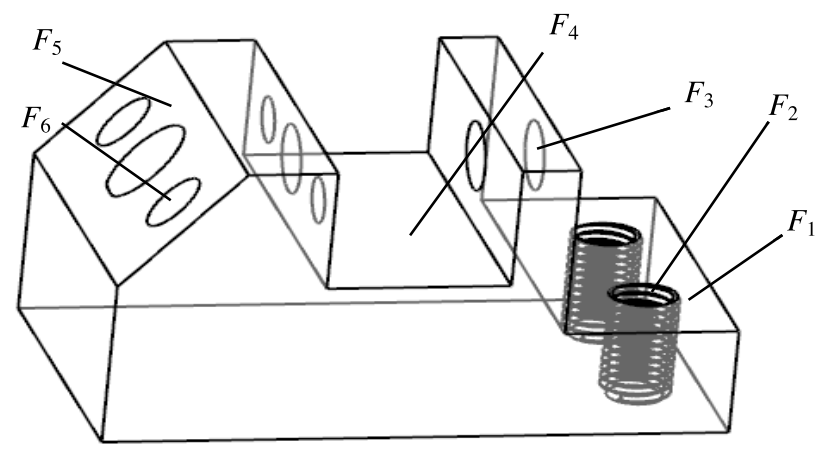

Fig. 1. An example part. 
Table 2. Operation selection for the example part.

\begin{tabular}{|c|c|c|c|c|}
\hline Features & Operations & Machines & Tools & TADs \\
\hline$F_{1}$ & Milling $\left(O P_{1}\right)$ & $M_{1}$ & $\mathfrak{d}_{1}$ & $-\mathscr{Q},-\mathscr{X}$ \\
\hline \multirow[t]{2}{*}{$F_{2}$} & $\operatorname{Drilling}\left(O P_{2}\right)$ & $M_{1}, M_{2}$ & $\mathscr{J}_{2}$ & $-g$ \\
\hline & Tapping $\left(\mathrm{OP}_{3}\right)$ & $M_{1}, M_{2}$ & $\mathfrak{d}_{3}$ & $-g$ \\
\hline \multirow[t]{2}{*}{$F_{3}$} & Drilling $\left(O P_{4}\right)$ & $M_{1}, M_{2}$ & $\mathscr{J}_{4}$ & $\mathscr{X}$ \\
\hline & Reaming $\left(O P_{5}\right)$ & $M_{1}, M_{2}$ & $\mathcal{D}_{5}$ & $\mathscr{X}$ \\
\hline$F_{4}$ & $\operatorname{Milling}\left(O P_{6}\right)$ & $M_{1}$ & $\mathcal{J}_{1}, \mathcal{J}_{6}$ & $-g$ \\
\hline$F_{5}$ & Milling $\left(O P_{7}\right)$ & $M_{1}$ & $\mathscr{J}_{1}, \mathcal{J}_{6}$ & $-g,+\mathscr{y}$ \\
\hline \multirow[t]{2}{*}{$F_{6}$} & Drilling $\left(O P_{8}\right)$ & $M_{1}, M_{2}$ & $\mathfrak{d}_{\tau}$ & $+\mathscr{X}$ \\
\hline & Reaming $\left(O P_{9}\right)$ & $M_{1}, M_{2}$ & $\mathscr{T}_{8}$ & $+\mathscr{X}$ \\
\hline
\end{tabular}

In the above table, $M_{1}$ means vertical milling machine, $M_{2}$ means drilling machine, $T_{1}$ means mill cutter $1, T_{2}$ means drill 1, $T_{3}$ means tapping tool, $T_{4}$ means drill $2, T_{5}$ means reamer $1, T_{6}$ means mill cutter $2, T_{7}$ means drill 3 , and $T_{8}$ means reamer 2 . The alternative operation is as shown in table 3 according to the description of the formula (5):

Table 3. Description of the alternative operations.

\begin{tabular}{|c|c|c|c|c|c|}
\hline Numbers & Operation & $\begin{array}{l}\text { Alternative } \\
\text { Operation }\end{array}$ & Machines & Tools & TADs \\
\hline$O_{1}$ & $O P_{1}$ & $A O_{11}$ & $M_{1}$ & $\mathscr{J}_{1}$ & -9 \\
\hline $\mathrm{O}_{2}$ & $O P_{1}$ & $A O_{12}$ & $M_{1}$ & $\mathcal{E}_{1}^{\mathfrak{T}}$ & $-\mathscr{X}$ \\
\hline$O_{3}$ & $\mathrm{OP}_{2}$ & $A O_{21}$ & $M_{1}, M_{2}$ & $\mathcal{J}_{2}$ & -9 \\
\hline$O_{4}$ & $\mathrm{OP}_{3}$ & $A O_{31}$ & $M_{1}, M_{2}$ & $\mathscr{D}_{8}^{\mathfrak{T}}$ & -9 \\
\hline$O_{5}$ & $\mathrm{OP}_{4}$ & $A O_{41}$ & $M_{1}, M_{2}$ & $\mathscr{J}_{4}$ & $\mathscr{X}$ \\
\hline$O_{6}$ & $O P_{5}$ & $A O_{51}$ & $M_{1}, M_{2}$ & $\mathscr{D}_{5}$ & $-\mathscr{X}$ \\
\hline$O_{7}$ & $O P_{6}$ & $A O_{61}$ & $M_{1}$ & $\mathcal{E}_{1}, \mathcal{J}_{6}$ & -9 \\
\hline$O_{8}$ & $O P_{7}$ & $A O_{71}$ & $M_{1}$ & $\mathcal{E}_{1}, \mathcal{J}_{6}$ & - g \\
\hline$O_{9}$ & $O P_{7}$ & $A O_{72}$ & $M_{1}$ & $\mathcal{C}_{1}, \mathcal{C}_{6}^{\mathcal{T}}$ & $+\mathscr{Y}$ \\
\hline$O_{10}$ & $O P_{8}$ & $A O_{81}$ & $M_{1}, M_{2}$ & $\mathscr{J}_{\tau}$ & $+\mathscr{X}$ \\
\hline$O_{11}$ & $O P_{9}$ & $A O_{91}$ & $M_{1}, M_{2}$ & $\mathcal{E}_{\tau}$ & $+\mathscr{X}$ \\
\hline
\end{tabular}

Along the same tool access direction of adjacency features, alternative machining operations $A O_{11}$ and $A O_{21}$ have the preference constraint relationship. Preference constraint relations in the machining operations are as follows: $O P_{1}$ is prior to $O P_{2}$ and $O P_{3}, O P_{2}$ is prior to $O P_{3}, O P_{4}$ is prior to $O P_{5}, O P_{4}$ and $O P_{5}$ are prior to $O P_{6}, O P_{8}$ is prior to $O P_{9}$, 
and $O P_{8}$ and $O P_{9}$ are prior to $O P_{7}$. When there are preference constraint relations between machining operations, then the alternative machining operation also has the corresponding preference constraint relation; according to formula (4), preference constraint matrix is as shown in figure 2.

\begin{tabular}{|c|c|c|c|c|c|c|c|c|c|c|c|}
\hline & $O_{1}$ & $\mathrm{O}_{2}$ & $O_{3}$ & $O_{4}$ & $O_{5}$ & $O_{6}$ & $O_{7}$ & $O_{8}$ & $O_{9}$ & $O_{10}$ & $O_{11}$ \\
\hline$O_{1}$ & {$[0$} & -1 & 1 & 1 & 0 & 0 & 0 & 0 & 0 & 0 & \\
\hline $\mathrm{O}_{2}$ & -1 & 0 & 1 & 1 & 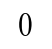 & 0 & 0 & 0 & 0 & 0 & \\
\hline$U$ & 0 & 0 & 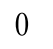 & 1 & 0 & 0 & 0 & 0 & 0 & 0 & 0 \\
\hline$U$ & 0 & 0 & 0 & 0 & 0 & 0 & 0 & 0 & 0 & 0 & 0 \\
\hline$O_{\xi}$ & 0 & 0 & 0 & 0 & 0 & 1 & 1 & 0 & 0 & 0 & 0 \\
\hline$O_{6}$ & 0 & 0 & 0 & 0 & 0 & 0 & 1 & 0 & 0 & 0 & 0 \\
\hline$\Omega$ & 0 & 0 & 0 & 0 & 0 & 0 & 0 & 0 & 0 & 0 & 0 \\
\hline Q & 0 & 0 & 0 & 0 & 0 & 0 & 0 & 0 & -1 & 0 & 0 \\
\hline & 0 & 0 & 0 & 0 & 0 & 0 & 0 & -1 & 0 & 0 & 0 \\
\hline & 0 & 0 & 0 & 0 & 0 & 0 & 0 & 1 & 1 & 0 & 1 \\
\hline & Lo & 0 & 0 & 0 & 0 & 0 & 0 & 1 & 1 & 0 & - \\
\hline
\end{tabular}

Fig. 2. The precedence constraint matrix.

According to the definition of the above alternative machining operator precedence constraint matrix $\mathrm{C}$, if there is $C_{i j}=C_{j i}=-1$, then $C_{i j}=C_{j i}=0$; get the matrix $\mathrm{C}^{\prime}$. Alternative machining operations 0 are divided into three types on the basis of $\mathrm{C}^{\prime}$ : independent alternative machining operations, priority alternative machining operations, and subsequent alternative machining operations; they are, respectively, defined as follows.

Definition 1 Independent alternative machining operations set $0_{I}$. If alternative machining operations $O_{i}$ is in precedence constraint matrix, then there is a relation $\sum_{i=1}^{N} C_{i j}=\sum_{j=1}^{N} C_{j i}=0$, indicating that the alternative machining operation has no precedence constraint relationship with other alternative operations, which is the independent alternative machining operations.

Definition 2 Precedence alternative machining operations set $0_{P}$. If alternative machining operation $O_{i}$ is in precedence constraint matrix, then there is a relation $\sum_{i=1}^{N} C_{i j}=0$ and $\sum_{j=1}^{N} C_{j i} \neq 0$, revealing that $O_{i}$ is precedence alternative machining operation of one or more alternative machining operations.

Definition 3 Subsequent alternative machining operations set $0_{s}$. If alternative processing operation $O_{i}$ is in precedence constraint matrix, then there is a relation $\sum_{i=1}^{N} C_{i j} \neq 0$, indicating that $O_{i}$ begins to machine after one or more alternative machining operations have finished, which is the subsequent alternative processing operation.

According to the above definition, $O_{1}, O_{2}, O_{5}$ and $O_{10}$ belong to precedence alternative machining operations set $0_{P}$, while the others belong to the subsequent alternative machining operations set $0_{S}$; there is no independent alternative machining operations set $0_{I}$. The precedence alternative machining operations set $0_{P}$ and independent alternative machining operations set $0_{I}$ belong to the current feasible alternative machining operations. So the definition of feasible alternative machining operation can be defined as follows.

Definition 4 feasible alternative machining operations set $0_{F}$. If alternative machining operation $O_{i}$ is in precedence constraint matrix, then there is a relation $\sum_{i=1}^{N} C_{i j}=0$, revealing that $O_{i}$ is precedence alternative machining operation or independent alternative machining operations, which is the feasible alternative processing operation. 


\section{GENERATION OF DYNAMIC FEASIBLE ALTERNATIVE MACHINING OPERATIONS SET}

When planning the flexible manufacturing route, the chosen alternative machining operations will not be chosen again, which belong to Tabu alternative machining operations set. And when a certain machining operation has more alternative machining operations, the other alternative machining operations will not be chosen again after one of the alternative machining operations is chosen, which also belongs to Tabu alternative machining operations set. Suppose Tabu alternative processing operation list is $\mathrm{Tab}_{t}(0)$, then the feasible alternative processing operation list is $\mathrm{Fea}_{t+1}\left(\mathrm{O}_{F}\right)=\left(\mathrm{Fea}_{t}\left(\mathrm{O}_{F}\right)-\mathrm{Tab}_{t}(0)\right)$. Since Tabu alternative processing operation list $\mathrm{Tab}_{t}(0)$ is updated dynamically with the selection of each step, feasible alternative machining operation list $\mathrm{Fea}_{t+1}\left(0_{F}\right)$ is also dynamically updated.

The selected nodes are all machining operations selected from current feasible alternative processing operations list. It assumes the current selected alternative machining operation is $O_{i}$, and the alternative machining operations set of process route is $0_{R}$. According to the preceding definition, there are two ways to achieve the dynamic update of $0_{F}$; one is to update dynamically $0_{s}$, and combine $0_{s}$ with $0_{R}$, and generate $\mathrm{Tab}_{t}(0)$ to realize the dynamic update of $\mathrm{Fea}_{t+1}\left(\mathrm{O}_{F}\right)$. The other method is that the diagonal values of the alternative machining operation choose to enter $0_{R}$ in each step. And other alternative machining operations belong to the same machining operations and are all set to constant according to the definition of $0_{F}$, so that they do not meet the definition that each column sum is zero and realize the dynamic update of $0_{F}$. The second method is employed in this paper, and then the generating process of current dynamic update feasible alternative processing operations set is as shown in figure 3 .

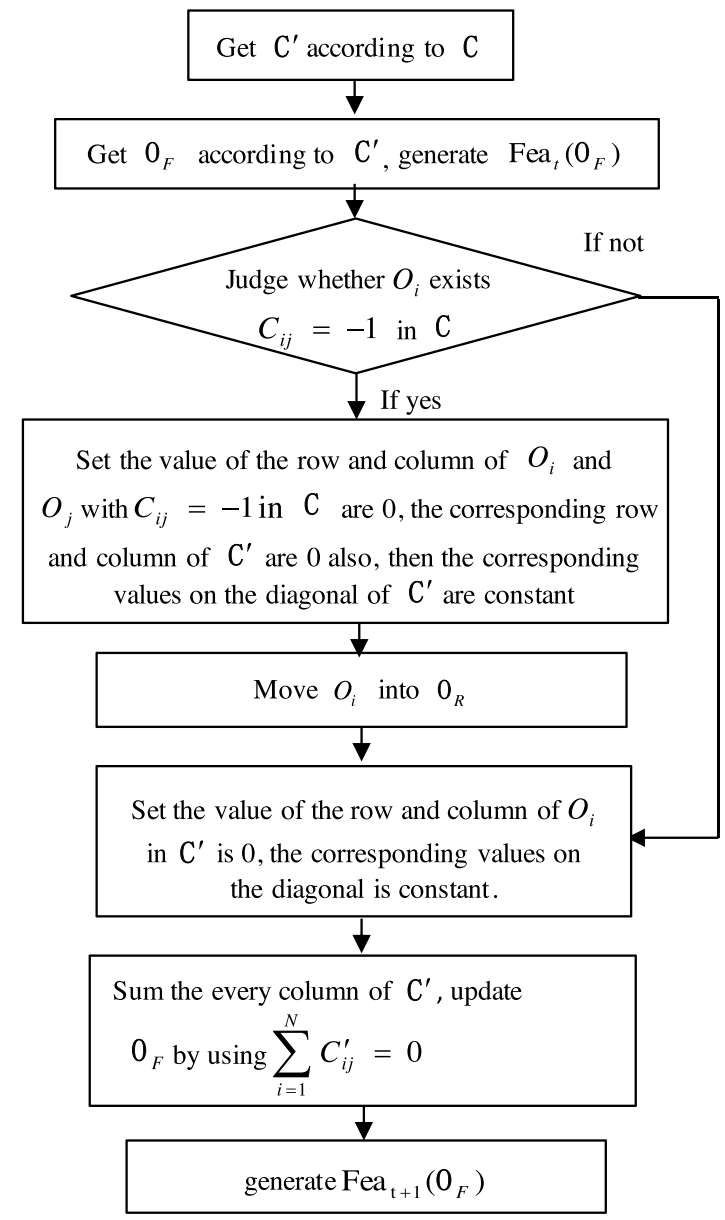

Fig. 3. Generating process of feasible alternative processing operation list. 
The steps generated by the above feasible alternative machining operations list are explained as follows:

Step 1: If there is $C_{i j}=C_{j i}=-1$ in alternative processing operator precedence constraints matrix $\mathrm{C}$, then set $C_{i j}=C_{j i}=0$, and get matrix $\mathrm{C}^{\prime}$.

Step 2: Using definition 4 , we can get feasible alternative machining operations set $0_{F}$ based on matrix $C^{\prime}$; then feasible alternative machining operations list $\mathrm{Fea}_{t}\left(0_{F}\right)$ can be gotten in time $t$.

Step 3: Determine whether $O_{i}$ exists $C_{i j}=-1$ in C; if no, go to Step 6.

Step 4: If yes, set the value of the row and column of $O_{i}$ and $O_{j}$ with $C_{i j}=-1$ in $\mathrm{C}$ and $\mathrm{C}^{\prime}$ as 0 ; the corresponding values on the diagonal of $\mathrm{C}^{\prime}$ are set to constant;

Step 5: Move $O_{i}$ into process route set $0_{R}$;

Step 6: Set the values of the row and column of $O_{i}$ in $\mathrm{C}^{\prime}$ as 0 ; the corresponding row and column of $\mathrm{C}^{\prime}$ are 0 as well; then the corresponding values on the diagonal of $\mathrm{C}^{\prime}$ are constant;

Step 7: Sum every column of $C^{\prime}$, and update feasible alternative processing operation set $0_{F}$ under the condition of $\sum_{i=1}^{N} C_{i j}^{\prime}=0$; then feasible alternative machining operations list $\mathrm{Fea}_{t+1}\left(0_{F}\right)$ can be gotten in time $t+1$.

The selection process of a process route of parts shown in figure 1 is shown in figure 4 .

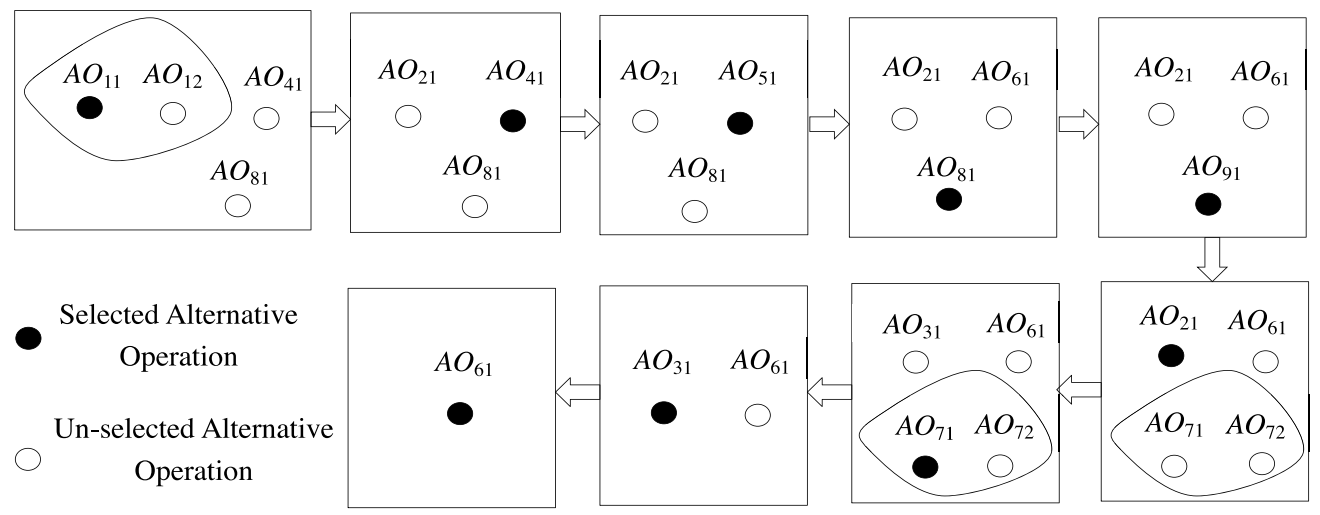

Fig. 4. Selected alternative operation for the example part.

Process route selection of the above parts is carried out in feasible alternative processing operation every time the alternative machining operations are updated dynamically. At the same time, it is clear that the scale of the solving problem is greatly shrunk after the search space limit of each step of the process planning.

\section{OPTIMIZATION OBJECTIVE OF FLEXIBLE PROCESS PLANNING}

The flexible process planning problem is generally described as follows: in view of the all feasible processing route of machined parts, choose from one of them, which optimizes some indicators. In the existing study, optimization goal based on machining time and cost are usually used (Liu et al., 2013; Blanch et al., 2011; Ciurana et al., 2008). The optimization goal based on the manufacturing cost is mainly considered in this paper, which will include the following five aspects: cost of machine tools, tooling cost, cost of machine tool changing, cost of tools changing, and cost of the clamping changing; the specific calculation process are described as follows:

(1) Machine tools cost of the total parts processing (TMC)

$$
T M C=\sum_{i=1}^{n} M_{j} C_{i}, \quad j=O P[i] . \text { machine_id }
$$


$M_{j} C_{i}$ is the manufacturing cost of machine tool that the $j$-th machine tools in the $i$-th machining operations need, and $n$ is the number of machining operations.

(2) The total tooling cost of parts processing (TTC)

$T T C=\sum_{i=1}^{n} T_{j} C_{i}, \quad j=$ OP[i].tool_id

$T_{j} C_{i}$ is the tooling cost that the $\mathrm{j}$-th tools in the $\mathrm{i}$-th machining operation need.

(3) The total cost of machine tool changing (TMCC)

$T M C C=M C C \times N M C$

$M C C$ is the cost that machine tools need; we can consider that every time the cost of the machine tool transformation is the same. $N M C$ is the times of machine tool transformation, which can be calculated by formula (9) and formula (10).

$$
\begin{aligned}
& N M C=\sum_{i=1}^{n-1} \Omega 1\left(M_{j}, M_{k}\right), \quad j=O P[i] . m a c h i n e \_i d \quad k=O P[i+1] . m a c h i n e \_i d \\
& \Omega 1(X, Y)= \begin{cases}1, & X \neq Y \\
0, & X=Y\end{cases}
\end{aligned}
$$

(4) The total cost of tools changing (TTCC)

The machining operations of the two adjacent parts do not need to transform tool only when using the same tool and machine tool. Otherwise, the tools need to be changed.

$T T C C=T C C \times N T C$

TCC is the cost of tools changing once. NTC is the times of tools changing, which can be calculated by formulas (12) and (13).

$$
\begin{aligned}
& N T C=\sum_{i=1}^{n-1} \Omega 2\left(\Omega 1\left(M_{j}, M_{k}\right), \Omega 1\left(T_{l}, T_{m}\right)\right) \\
& j=O P[i] . m a c h i n e \_i d, k=O P[i+1] . m a c h i n e \_i d \\
& l=O P[i] . t o o l \_i d, m=O P[i+1] . t o o l \_i d \\
& \Omega 2(X, Y)=\left\{\begin{array}{l}
0, X=Y=0 \\
1, \text { ortherwise }
\end{array}\right.
\end{aligned}
$$

(5) The set-up changing cost of parts (TSCC)

The machining operations of the two adjacent parts can be thought as they do not need set-up changing only when they use the same tool and the same feed direction. Otherwise, set-up changing needs to be done once.

$T S C C=(N S C+1) \times S C C$

$S C C$ is the cost of set-up changing once. NSC is the times of production preparation, which can be calculated by formulas (15) and (16).

$$
\begin{aligned}
& N S C=\sum_{i=1}^{n} \Omega 2\left(\Omega 1\left(M_{j}, M_{k}\right), \Omega 1\left(T A D_{l}, T A D_{m}\right)\right) \\
& j=O P[i] . m a c h i n e \_i d, k=O P[i+1] . m a c h i n e \_i d \\
& l=O P[i] . T A D \_i d, m=O P[i+1] . T A D \_i d
\end{aligned}
$$


$N S=N S C+1$

(6) The total weighted cost of parts $(T W C)$

The optimization goal based on the machining cost can be set to minimum total weighted cost, which is shown as formula (17):

$$
\operatorname{Min} T W C=w_{1} \times T M C+w_{2} \times T T C+w_{3} \times T M C C+w_{4} \times T T C C+w_{5} \times T S C C
$$

\section{A NOVEL TWO-STAGE ACO ALGORITHM FOR FPP}

The ACO algorithm is a population-based heuristic, which simulates the foraging behavior of ants and develops mechanisms of cooperation and learning to solve optimization problems (Dorigo et al., 1996). In an ant colony, ants lay pheromone trails on the path while foraging to share information with other ants and direct them toward food sources. An isolated ant may move randomly and lay pheromone trail over traversed path. An ant encountering a previously laid trail can apply a probabilistic approach, which favors the path with the highest pheromone trail to decide where to go, thus reinforcing the trail with its own pheromone. This forms a positive feedback, enabling rapid discovery of food source. Eventually, with the cooperation among ants, the shortest route from the colony to food source can be established. ACO uses the similar cooperation mechanism to search for good solutions to optimization problems.

The flexible process planning problem is a non-deterministic polynomial complete (NP-Complete) problem (Liu et al., 2013). Due to the existence of operation precedence constraints in the problem, infeasible solutions often occur when using heuristics (e.g., GA, ACO, etc.) to solve the problem. To ensure the feasibility of the process plans, additional adjustments need to be done. The adjustment methods that prevail in the literature include constraint adjustment method (Li et al., 2002), penalty function method, and restricting the search space. Due to lack of prior information, when dealing with complex precedence constraints, constraint adjustment methods may lead to a very slow convergence of the algorithm, and penalty function method mainly applies when there is a clear boundary between feasible and infeasible regions. Hence, this paper utilizes the adjustment method of restricting the search space and develops a novel ACO algorithm integrated with a dynamic adjustment of the alternative machining operations set. The proposed algorithm consists of two stages: i) the first stage is for constraint-based operation selection and sequencing; ii) the second stage aims at allocating resources (i.e., machines and tools) for the selected and sequenced operations obtained from the first stage, taking each alternative machine-tool combination for an operation as a subnode that subordinates to that operation node.

\section{ANT COLONY AND TRANSITION PROBABILITY}

Assume that there are $N$ operations. Take each operation as a particular node in the graph. An edge $\left(O_{i}, O_{j}\right)$ represents that operation $O_{j}$ be processed right after $O_{i}$. Let $m$ be the total number of ants in the colony, $b_{i}(t)$ be the number of ants situated at operation node $O_{i}$ at time $t\left(\sum_{i=1}^{N} b_{i}(t)=m\right)$, and $\tau_{i j}(t)$ be the intensity of pheromone trail on edge $\left(O_{i}, O_{j}\right)$ at time $t$. Take $\Gamma=\left\{\tau_{i j}(t) \mid 0_{i}, 0_{j} \subset 0\right\}$ as the set of pheromone trail intensity for all the alternative edges at time $t$. Assume that the initial intensities of pheromone trail on each edge are equal, and $\tau_{i j}(0)=$ const.

Individual ants lay pheromone trails on the traversed edges and choose their next operation node with respect to probabilities that depend on pheromone trails and the attractiveness of alternative options (i.e., next operation). The transition probability for ant $k(k=1,2, \cdots, m)$ at operation node $O_{i}$ to move to node $O_{j}$ for the next step is defined as

$$
P_{i j}^{k}(t)=\left\{\begin{array}{cc}
\frac{\left[\tau_{i j}(t)\right]^{\alpha}\left[\eta_{i k}(t)\right]^{\beta}}{\sum_{\mathrm{s} \in \mathrm{Fea}_{t}(0)}\left[\tau_{i s}(t)\right]^{\alpha}\left[\eta_{i s}(t)\right]^{\beta}} & s \in \mathrm{Fea}_{t}(0) \\
0 & \text { otherwise }
\end{array}\right.
$$

where $\mathrm{Fea}_{t}(0)$ represents the set of all the alternative operation nodes for the next step, and parameters $\alpha$ and $\beta$ weigh the relative importance of trail versus attractiveness. $\eta_{i j}(t)$ is a heuristic function that evaluates the attractiveness 
of edge $\left(O_{i}, O_{j}\right)$, which depends on the production cost of the edge, as shown in Eq. (19). The less an edge cost is, the more attractive it is; hence there is a higher probability for ants following it.

$$
\eta_{i k}(t)=\frac{1}{C_{i j}}
$$

\section{PHEROMONE UPDATING}

At each iteration, individual ants change pheromone trail intensity by laying their own pheromone trails on the traversed edges. Pheromones are updated by the following rule:

$$
\begin{aligned}
& \tau_{i j}(t+N)=(1-\rho) \cdot \tau_{i j}(t)+\Delta \tau_{i j}(t) \\
& \Delta \tau_{i j}(t)=\sum_{k=1}^{m} \Delta \tau_{i j}^{k}(t) \\
& \Delta \tau_{i j}^{k}(t)=\left\{\begin{array}{cc}
Q / T W C & \text { if ant k traverses edge }\left(O_{i}, O_{j}\right) \\
0 & \text { otherwise }
\end{array}\right.
\end{aligned}
$$

where $\rho \in[0,1)$ is the pheromone evaporation rate, and $1-\rho$ stands for the pheromone persistence factor. $\Delta \tau_{i j}(t)$ is the pheromone increment of edge $\left(O_{i}, O_{j}\right)$, and $\Delta \tau_{i j}^{k}(t)$ refers to the quantity of pheromone laid on edge $\left(O_{i}, O_{j}\right)$ by ant $k(k=1,2, \cdots, m) . Q$ is a constant and $T W C$ is the total weighted cost defined in Eq. (17). Assume that $\Delta \tau_{i j}(0)=0$.

To better explore the search space and prevent premature convergence, a lower bound $\tau_{\min }$ and an upper bound $\tau_{\max }$ are imposed on pheromone trails, preventing the relative differences between pheromone trails from becoming too extreme. Use Eq. (24) to adjust trails within $\left[\tau_{\min }, \tau_{\max }\right]$.

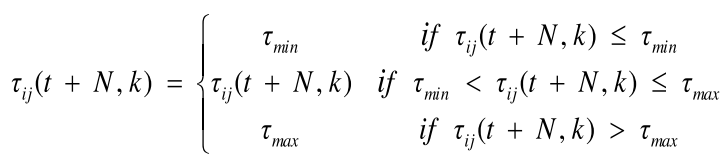

\section{OVERALL PROCEDURE OF THE PROPOSED ALGORITHM FOR FPP}

The main idea of the proposed algorithm is as follows: First randomly place each ant on an operation node from the feasible alternative machining operations list $\mathrm{Fea}_{0}\left(\mathrm{O}_{F}\right)$; update the precedence constraint matrix and the feasible alternative machining operations set $0_{F}$; select the next operation node from the updated feasible alternative machining operations list $\mathrm{Fea}_{t}\left(0_{F}\right)$. Synthesize the transition probability to alternative operations; repeat the previous two steps until a feasible complete process plan has been produced. Through iterations of ants searching, the process plan with the least cost can be obtained.

Step 0 . Specify the parameters such as the total number of ants $m$, the pheromone evaporation rate $\rho$, weights $\alpha$ and $\beta$, and the maximal iteration number $N_{c_{\max }}$. Set $\tau_{i j}(0)=$ const and $\Delta \tau_{i j}(0)=0(i, j=1,2, \cdots, N)$. Set $t=0$ and the iteration number $N_{c}=1$.

\section{Stage 1 Precedence constraint-based operation selection}

Step 1.1 Set the index of ant $k=1$.

Step 1.2 Obtain the feasible alternative machining operations set $0_{F}$, and initial feasible alternative machining operations list $\mathrm{Fea}_{0}\left(0_{F}\right)$.

Step 1.3 Randomly place ant $k$ on an operation node from $\mathrm{Fea}_{0}\left(0_{F}\right)$. Set $t=t+1$. Update $\mathrm{Fea}_{t}\left(0_{F}\right)$ according to the dynamic adjustment method of the alternative operations set. 
Step 1.4 Compute $\eta_{i k}(t)$ and $P_{i j}^{k}(t)$ according to Eqs. (19)-(20). With the transition probability $P_{i j}^{k}(t)$, select the next operation node from $\mathrm{Fea}_{t}(0)$ for ant $k$.

Step 1.5 Set $t=t+1$. Update Fea $(0)$.

Step 1.6 If there is no operation left in $\mathrm{Fea}_{t}(0)$, execute Stage 2. Otherwise, go to Step 1.4.

Stage 2 Machine and tool resource allocation

Step 2.1 Set the maximal cycle times $N_{m-\max }, N_{m}=N_{m}+1$.

Step 2.2 Randomly choose a machine from Machine_list and a tool from Tool_list for the first selected operation $O_{i}$ in the process plan (i.e., the traversed route of ant $k$ ) generated in Stage 1. Record the machine ID and the tool ID.

Step 2.3 Choose the machine-tool combination for the next operation with the least changeover cost when switching from the previous operation. This step continues until all the operations in the process plan have been allocated with machine and tool resource.

Step 2.4 Compute the total weighted cost $T W C$ for the process plan generated by ant $k$.

Step 2.5 Find the minimum $T W C$ in all cycle times, and save the process route information of manufacturing resource selection.

Step 2.6 Set $k=k+1$. If $k<m$, and return to Step 1.2. Otherwise, execute Step 2.7.

Step 2.7 Find the best process plan with the least TWC among all the $m$ process plans generated by these $m$ ants.

Step 2.8 Update the pheromones according to Eqs. (20)-(24). Set $N_{c}=N_{c}+1$.

Step 3 If $N_{c} \geq N_{c_{\max }}$, terminate the algorithm and output the best process plan with the minimum TWC. Otherwise, empty the alternative operations set, and return to Stage 1.

\section{CASE STUDY}

The algorithm proposed in this paper is verified by the part shown in figure 5 (Li et al., 2002); firstly, the conditions set are as follows: (a) all the machine tools and cutting tools are available; set the value of $w_{1} \sim w_{5}$ in objective function (17) to be 1. (b) All the machine tools and cutting tools are available, in objective function (17), $w_{2}=w_{5}=0$, $w_{1}=w_{3}=w_{4}=1$.

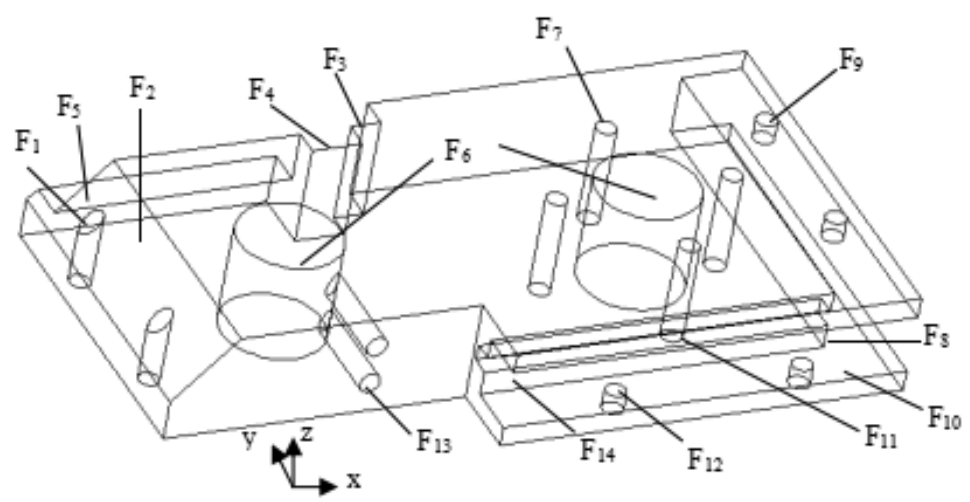

Fig. 5. Geometric figure of Instance parts.

The above parts have 14 manufacturing features. The relevant resources and costs of machines and tools are shown in tables 4-5, and the related information of machining operations are shown in table 6. Suppose the $M C C$ 
cost of machines changing once is 300 , the $S C C$ cost of tools changing once is 120 , and the $S C C$ cost of starting the transformation once is 15 .

Table 4. Machine resources and costs.

\begin{tabular}{ccc}
\hline Machines no. & Types & $m c_{i}$ \\
\hline 1 & Drill machine & 10 \\
2 & Milling machine & 35 \\
3 & Three-axis vertical milling machine & 60 \\
\hline
\end{tabular}

Table 5. Tool resources and costs.

\begin{tabular}{ccc}
\hline Tools no. & Types & $m c_{i}$ \\
\hline 1 & Drill 1 & 3 \\
2 & Drill 2 & 3 \\
3 & Reamer & 8 \\
4 & Boring tool & 15 \\
5 & Milling cutter 1 & 10 \\
6 & Milling cutter 2 & 15 \\
7 & Slot cutter & 10 \\
8 & Chamfer tool & 10 \\
\hline
\end{tabular}

Table 6. Description of the alternative operations.

\begin{tabular}{|c|c|c|c|c|c|c|c|}
\hline Numbers & Features & $\begin{array}{c}\text { Feature } \\
\text { descriptions }\end{array}$ & Operations & $\begin{array}{l}\text { Alternative } \\
\text { Operations }\end{array}$ & Machines & Tools & $\mathrm{TAD}$ \\
\hline$O_{1}$ & $F_{1}$ & Two holes & $O P_{1}$ & $A O_{11}$ & $M_{1}, M_{2}, M_{3}$ & $\mathcal{D}_{1}^{\mathcal{T}}$ & $+g$ \\
\hline $\mathrm{O}_{2}$ & $F_{1}$ & & $O P_{1}$ & $A O_{12}$ & $M_{1}, M_{2}, M_{3}$ & $\mathscr{D}_{1}$ & $-g$ \\
\hline$O_{3}$ & $F_{2}$ & A chamfer & $\mathrm{OP}_{2}$ & $A O_{21}$ & $M_{2}, M_{3}$ & $\mathscr{J}_{8}$ & $-\mathscr{X}$ \\
\hline $\mathrm{O}_{4}$ & $F_{2}$ & & $\mathrm{OP}_{2}$ & $A O_{22}$ & $M_{2}, M_{3}$ & $\mathscr{J}_{8}$ & $+\mathscr{y}$ \\
\hline$O_{5}$ & $F_{2}$ & & $\mathrm{OP}_{2}$ & $\mathrm{AO}_{23}$ & $M_{2}, M_{3}$ & $\mathcal{J}_{8}^{\mathfrak{T}}$ & $-\mathscr{Y}$ \\
\hline$O_{6}$ & $F_{2}$ & & $\mathrm{OP}_{2}$ & $A O_{24}$ & $M_{2}, M_{3}$ & $\mathscr{J}_{8}^{\mathfrak{T}}$ & $-\mathscr{P}$ \\
\hline$O_{7}$ & $F_{3}$ & A slot & $\mathrm{OP}_{3}$ & $A O_{31}$ & $M_{2}, M_{3}$ & $\mathcal{E}_{\tilde{F}^{\prime}}, \mathscr{J}_{6}$ & $+\mathscr{y}$ \\
\hline$O_{8}$ & $F_{4}$ & A slot & $\mathrm{OP}_{4}$ & $A O_{41}$ & $M_{2}$ & $\mathcal{D}_{5}, \mathscr{J}_{6}^{\mathcal{T}}$ & $+\mathscr{y}$ \\
\hline$O_{9}$ & $F_{5}$ & A step & $\mathrm{OP}_{5}$ & $A O_{51}$ & $M_{2}, M_{3}$ & $\mathcal{D}_{5}, \mathfrak{J}_{6}^{\mathcal{T}}$ & $+\mathscr{Y}$ \\
\hline
\end{tabular}




\begin{tabular}{|c|c|c|c|c|c|c|c|}
\hline$O_{10}$ & $F_{5}$ & & $O P_{5}$ & $A O_{52}$ & $M_{2}, M_{3}$ & $\mathscr{D}_{\tilde{J}^{\prime}}, \mathscr{J}_{6}$ & $-g$ \\
\hline$O_{11}$ & $F_{6}$ & Two holes & $O P_{6}$ & $A O_{61}$ & $M_{1}, M_{2}, M_{3}$ & $\mathfrak{J}_{2}$ & $+g$ \\
\hline$O_{12}$ & $F_{6}$ & & $O P_{6}$ & $A O_{62}$ & $M_{1}, M_{2}, M_{3}$ & $\mathcal{J}_{2}$ & $-g$ \\
\hline$O_{13}$ & $F_{7}$ & Four holes & $O P_{7}$ & $A O_{71}$ & $M_{1}, M_{2}, M_{3}$ & $\mathscr{D}_{1}$ & $+g$ \\
\hline$O_{14}$ & $F_{7}$ & & $O P_{7}$ & $A O_{72}$ & $M_{1}, M_{2}, M_{3}$ & $\mathcal{D}_{1}$ & $-g$ \\
\hline$O_{15}$ & $F_{8}$ & A slot & $O P_{8}$ & $A O_{81}$ & $M_{2}, M_{3}$ & $\mathcal{D}_{\tilde{5}^{\prime}}, \mathcal{J}_{6}$ & $+\mathscr{X}$ \\
\hline$O_{16}$ & $F_{9}$ & Two holes & $O P_{9}$ & $A O_{91}$ & $M_{1}, M_{2}, M_{3}$ & $\mathcal{D}_{1}^{\mathfrak{T}}$ & $-g$ \\
\hline$O_{17}$ & $F_{10}$ & A slot & $O P_{10}$ & $A O_{101}$ & $M_{2}, M_{3}$ & $\mathcal{D}_{\tilde{5}^{\prime}} \mathcal{D}_{6}$ & $-y$ \\
\hline$O_{18}$ & $F_{11}$ & A slot & $O P_{11}$ & $A O_{111}$ & $M_{2}, M_{3}$ & $\mathcal{D}_{\tilde{5}}, \mathcal{C}_{6}$ & $-\mathscr{y}$ \\
\hline$O_{19}$ & $F_{12}$ & Two holes & $O P_{12}$ & $A O_{121}$ & $M_{1}, M_{2}, M_{3}$ & $\mathscr{D}_{1}$ & $+g$ \\
\hline$O_{20}$ & $F_{12}$ & & $O P_{12}$ & $A O_{122}$ & $M_{1}, M_{2}, M_{3}$ & $\mathscr{D}_{1}$ & -9 \\
\hline$O_{21}$ & $F_{13}$ & A step & $O P_{13}$ & $A O_{131}$ & $M_{2}, M_{3}$ & $\mathcal{D}_{\tilde{5}^{\prime}}, \mathcal{D}_{6}$ & $-\mathscr{X}$ \\
\hline$O_{22}$ & $F_{13}$ & & $O P_{13}$ & $A O_{132}$ & $M_{2}, M_{3}$ & $\mathcal{D}_{\tilde{5}}, \mathcal{J}_{6}$ & $-\mathscr{y}$ \\
\hline$O_{23}$ & $F_{14}$ & Two holes & $O P_{14}$ & $A O_{141}$ & $M_{1}, M_{2}, M_{3}$ & $\mathcal{D}_{1}$ & $-\mathscr{y}$ \\
\hline
\end{tabular}

In the literature (Li et al., 2004) and (Li et al., 2013), preference sequence constraints between each feature are shown in table 7.

Table 7. Machining constraints and interaction.

\begin{tabular}{ccc}
\hline Constraints & Descriptions & Hard or soft \\
\hline Tool interactions & $F_{1}$ should be prior to $F_{2}$ & Hard \\
Datum interactions & $F_{6}$ should be prior to $F_{7}$ & Hard \\
& $F_{10}$ should be prior to $F_{11}$ & \\
& $F_{13}$ should be prior to $F_{14}$ & \\
Thin-wall interactions & $F_{9}$ should be prior to $F_{8}$ & Soft \\
& $F_{12}$ should be prior to $F_{10}$ & \\
Material removal interactions & $F_{8}$ should be prior to $F_{9}$ & Soft \\
& $F_{10}$ should be prior to $F_{12}$ & \\
\hline
\end{tabular}

In the above machining precedence constraints, the feature $F_{1}$ has two alternative machining operations; when the feature $F_{1}$ uses $A O_{11}$ to machine, then the relation $F_{1}$ should be prior to $F_{2}$ which may be not established. Mainly consider the above Hard constraints of machining precedence constraints; the precedence constraint matrix is gotten as shown in figure 6 . 


\begin{tabular}{|c|c|c|c|c|c|c|c|c|c|c|c|c|c|c|c|c|c|c|c|c|c|c|c|}
\hline$O_{1}$ & $\begin{array}{l}O_{1} \\
0\end{array}$ & $\begin{array}{l}\mathrm{O}_{2} \\
-1\end{array}$ & $\begin{array}{c}0_{3} \\
0\end{array}$ & $\begin{array}{c}O_{4} \\
0\end{array}$ & $\begin{array}{c}O_{5} \\
0\end{array}$ & $\begin{array}{c}O_{6} \\
0\end{array}$ & $\begin{array}{c}O_{7} \\
0\end{array}$ & 0 & $\begin{array}{c}O_{9} \\
0\end{array}$ & $\begin{array}{c}O_{10} \\
0\end{array}$ & $\begin{array}{c}O_{11} \\
0\end{array}$ & $\begin{array}{c}O_{12} \\
0\end{array}$ & $\begin{array}{c}O_{13} \\
0\end{array}$ & $\begin{array}{c}O_{14} \\
0\end{array}$ & $\begin{array}{c}O_{15} \\
0\end{array}$ & $\begin{array}{c}O_{16} \\
0\end{array}$ & $\begin{array}{c}O_{17} \\
0\end{array}$ & $\begin{array}{c}O_{18} \\
0\end{array}$ & $\begin{array}{c}O_{19} \\
0\end{array}$ & $\begin{array}{c}O_{20} \\
0\end{array}$ & $\begin{array}{c}O_{21} \\
0\end{array}$ & $\begin{array}{c}O_{22} \\
0\end{array}$ & 0 \\
\hline $\mathrm{O}_{2}$ & -1 & 0 & 1 & 1 & 1 & 1 & 0 & 0 & 0 & 0 & 0 & 0 & 0 & 0 & 0 & 0 & 0 & 0 & 0 & 0 & 0 & 0 & 0 \\
\hline $\mathrm{O}_{3}$ & 0 & 0 & 0 & -1 & -1 & -1 & 0 & 0 & 0 & 0 & 0 & 0 & 0 & 0 & 0 & 0 & 0 & 0 & 0 & 0 & 0 & 0 & 0 \\
\hline$O_{4}$ & 0 & 0 & -1 & 0 & -1 & -1 & 0 & 0 & 0 & 0 & 0 & 0 & 0 & 0 & 0 & 0 & 0 & 0 & 0 & 0 & 0 & 0 & 0 \\
\hline$O_{5}$ & 0 & 0 & -1 & -1 & 0 & 0 & 0 & 0 & 0 & 0 & 0 & 0 & 0 & 0 & 0 & 0 & 0 & 0 & 0 & 0 & 0 & 0 & 0 \\
\hline$O_{6}$ & 0 & 0 & -1 & -1 & -1 & 0 & 0 & 0 & 0 & 0 & 0 & 0 & 0 & 0 & 0 & 0 & 0 & 0 & 0 & 0 & 0 & 0 & 0 \\
\hline$O_{7}$ & 0 & 0 & 0 & 0 & 0 & 0 & 0 & 0 & 0 & 0 & 0 & 0 & 0 & 0 & 0 & 0 & 0 & 0 & 0 & 0 & 0 & 0 & 0 \\
\hline$O_{8}$ & 0 & 0 & 0 & 0 & 0 & 0 & 0 & 0 & 0 & 0 & 0 & 0 & 0 & 0 & 0 & 0 & 0 & 0 & 0 & 0 & 0 & 0 & 0 \\
\hline $\mathrm{O}_{9}$ & 0 & 0 & 0 & 0 & 0 & 0 & 0 & 0 & 0 & -1 & 1 & 1 & 0 & 0 & 0 & 0 & 0 & 0 & 0 & 0 & 0 & 0 & 0 \\
\hline$O_{10}$ & 0 & 0 & 0 & 0 & 0 & 0 & 0 & 0 & -1 & 0 & 1 & 1 & 0 & 0 & 0 & 0 & 0 & 0 & 0 & 0 & 0 & 0 & 0 \\
\hline$O_{11}$ & 0 & 0 & 0 & 0 & 0 & 0 & 0 & 0 & 0 & 0 & 0 & -1 & 0 & 0 & 0 & 0 & 0 & 0 & 0 & 0 & 0 & 0 & 0 \\
\hline$O_{12}$ & 0 & 0 & 0 & 0 & 0 & 0 & 0 & 0 & 0 & 0 & -1 & 0 & 0 & 0 & 0 & 0 & 0 & 0 & 0 & 0 & 0 & 0 & 0 \\
\hline$O_{13}$ & 0 & 0 & 0 & 0 & 0 & 0 & 0 & 0 & 0 & 0 & 0 & 0 & 0 & -1 & 0 & 0 & 0 & 0 & 0 & 0 & 0 & 0 & 0 \\
\hline$O_{14}$ & 0 & 0 & 0 & 0 & 0 & 0 & 0 & 0 & 0 & 0 & 0 & 0 & -1 & 0 & 0 & 0 & 0 & 0 & 0 & 0 & 0 & 0 & 0 \\
\hline$O_{15}$ & 0 & 0 & 0 & 0 & 0 & 0 & 0 & 0 & 0 & 0 & 0 & 0 & 0 & 0 & 0 & 0 & 0 & 0 & 0 & 0 & 0 & 0 & 0 \\
\hline$O_{16}$ & 0 & 0 & 0 & 0 & 0 & 0 & 0 & 0 & 0 & 0 & 0 & 0 & 0 & 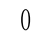 & ( & 0 & 0 & 0 & 0 & 0 & 0 & 0 & 0 \\
\hline$O_{17}$ & 0 & 0 & 0 & 0 & 0 & 0 & 0 & 0 & 0 & 0 & 0 & 0 & 0 & 0 & 0 & 0 & 0 & 1 & 0 & 0 & 0 & 0 & 0 \\
\hline$O_{18}$ & 0 & 0 & 0 & 0 & 0 & 0 & 0 & 0 & 0 & 0 & 0 & 0 & 0 & 0 & 0 & 0 & 0 & 0 & 0 & 0 & 0 & 0 & 0 \\
\hline$O_{19}$ & 0 & 0 & 0 & 0 & 0 & 0 & 0 & 0 & 0 & 0 & 0 & 0 & 0 & 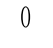 & 0 & 0 & 0 & 0 & 0 & -1 & 0 & 0 & 0 \\
\hline$O_{20}$ & 0 & 0 & 0 & 0 & 0 & 0 & 0 & 0 & 0 & 0 & 0 & 0 & 0 & 0 & 0 & 0 & 0 & 0 & -1 & 0 & 0 & 0 & 0 \\
\hline$O_{21}$ & 0 & 0 & 0 & 0 & 0 & 0 & 0 & 0 & 0 & 0 & 0 & 0 & 0 & 0 & 0 & 0 & 0 & 0 & 0 & 0 & 0 & -1 & 1 \\
\hline 0 & 0 & 0 & 0 & 0 & 0 & 0 & 0 & 0 & 0 & 0 & 0 & 0 & 0 & 0 & 0 & 0 & 0 & 0 & 0 & 0 & -1 & 0 & 1 \\
\hline $\mathrm{O}_{23}$ & 10 & 0 & 0 & 0 & 0 & 0 & 0 & 0 & 0 & 0 & 0 & 0 & 0 & 0 & 0 & 0 & 0 & 0 & 0 & 0 & 0 & 0 & 0 \\
\hline
\end{tabular}

Fig. 6. The precedence constraint matrix for the example part.

The setting parameters of ant colony algorithm are as follows: the number of ants $m=15, \alpha=1, \beta=2, \rho=0.1$, $N_{c_{\max }}=50, Q=3000, \tau_{i j}(0)=1$. The situations (a) and (b) of the instance parts, respectively, run 10 times; the optimum value of every generation is as shown in figures (6) and (7).

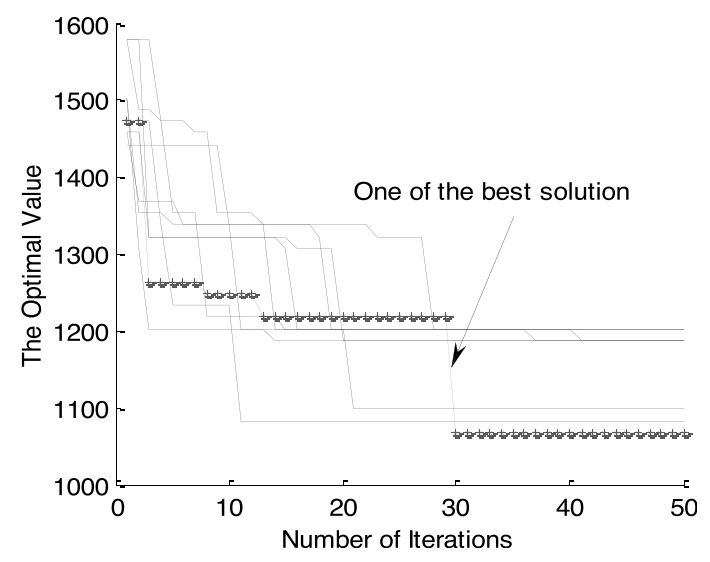

Fig. 6. Optimal value of each iteration in condition (a). 


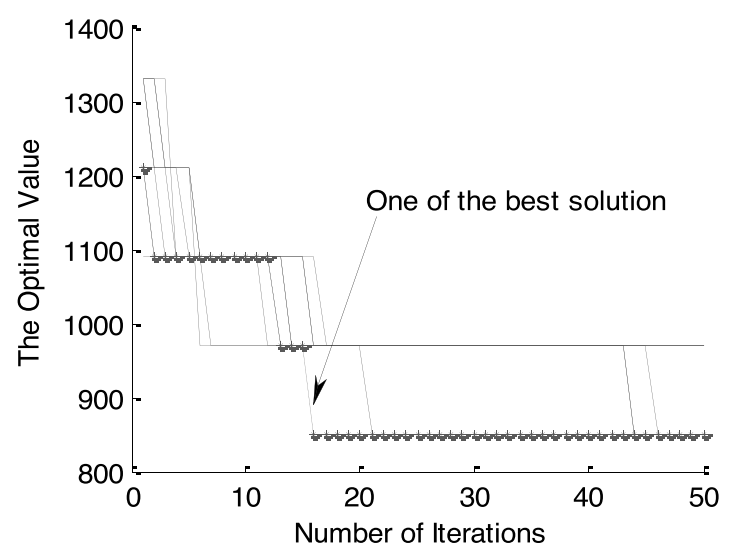

Fig. 7. Optimal value of each iteration in condition (b).

In the result of condition (a), TWC(1083) occurs 1 time, TWC(1098) occurs 1 time, TWC(1113) occurs 1 time, TWC(1188) occurs 4 times, and TWC(1203) occurs 3 times. In the result of condition (b), TWC(850) occurs 6 times, and TWC(970) occurs 4 times. The flexible precedence constraint problems are not considered in this paper, which will violate flexible constraint added to the total cost in the way of penalty cost. In this instance, there are two pairs of soft interactions shown in table 7: (1) $F_{9}$ should be prior to $F_{8}$ and $F_{8}$ should be prior to $F_{9}$. (2) $F_{12}$ should be prior to $F_{10}$ and $F_{10}$ should be prior to $F_{12}$. Therefore, in order to facilitate comparison with other algorithms, for any feasible process plan, the penalty cost should be 200 . The comparing results with other algorithms are shown in table 8 . The optimal solution under the conditions of (a) and (b) is shown in table (9) and table (10).

Table 8. The results compared for the example part.

\begin{tabular}{ccccccc}
\hline Condition & $\begin{array}{c}\text { Proposed } \\
\text { Algorithm }\end{array}$ & $\begin{array}{c}\text { Two-stage } \\
\text { ACO (Wang } \\
\text { et al., 2015) }\end{array}$ & $\begin{array}{c}\text { ACO } \\
\text { (Liu } \text { et al., 2013) })\end{array}$ & $\begin{array}{c}\text { Genetic } \\
\text { Algorithm } \\
\text { (Li } \text { et al., 2002) }\end{array}$ & $\begin{array}{c}\text { Simulated } \\
\text { annealing } \\
\text { (Li } \text { et al., 2002) }\end{array}$ & $\begin{array}{c}\text { Tabu search } \\
\text { (Li } \text { et al., 2014) }\end{array}$ \\
\end{tabular}

(a)

$\begin{array}{crrrrrr}\text { Mean } & 1363.9 & 1329.0 & 1329.5 & 1611.0 & 1373.5 & 1342.0 \\ \text { Maximum } & 1403.0 & 1348.0 & 1343.0 & 1778.0 & 1518.0 & 1378.0 \\ \text { Minimum } & 1283.0 & 1328.0 & 1328.0 & 1478.0 & 1328.0 & 1328.0\end{array}$

(b)

\begin{tabular}{|c|c|c|c|c|c|c|}
\hline Mean & 1098.0 & 1170.0 & 1170.0 & 1482.0 & 1217.0 & 1194.0 \\
\hline Maximum & 1170.0 & 1170.0 & 1170.0 & 1650.0 & 1345.0 & 1290.0 \\
\hline Minimum & 1050.0 & 1170.0 & 1170.0 & 1410.0 & 1170.0 & 1170.0 \\
\hline
\end{tabular}

It is shown from table 8 that the minimum TWC(1083) is the best result among all of six algorithms under the condition of (a). The minimum TWC(1050) and the mean TWC(1098) are the best results among all of six algorithms under the condition of (b). In the literature [19], the number of iterations is 200; the number of ants is 25 . If the number of iterations is increased, this can increase the occurrence number of relatively small optimization values such as TWC(1083) and TWC(1098) under the condition of (a) and further reduce the average optimized. 
Table 9. One of the best flexible process planning under condition (a) for the example part.

\begin{tabular}{cccccccccccccccc}
\hline No. & $O_{7}$ & $O_{9}$ & $O_{8}$ & $O_{17}$ & $O_{18}$ & $O_{22}$ & $O_{15}$ & $O_{23}$ & $O_{16}$ & $O_{14}$ & $O_{1}$ & $O_{19}$ & $O_{3}$ & $O_{12}$ \\
Feature & $F_{3}$ & $F_{5}$ & $F_{4}$ & $F_{10}$ & $F_{11}$ & $F_{13}$ & $F_{8}$ & $F_{14}$ & $F_{9}$ & $F_{7}$ & $F_{1}$ & $F_{12}$ & $F_{2}$ & $F_{6}$ \\
A-Op. & $A O_{31}$ & $A O_{51}$ & $A O_{41}$ & $A O_{101}$ & $A O_{111}$ & $A O_{132}$ & $A O_{81}$ & $A O_{141}$ & $A O_{91}$ & $A O_{72}$ & $A O_{1}$ & $A O_{121}$ & $A O_{21}$ & $A O_{62}$ \\
Machine & $M_{2}$ & $M_{2}$ & $M_{2}$ & $M_{2}$ & $M_{2}$ & $M_{2}$ & $M_{2}$ & $M_{2}$ & $M_{2}$ & $M_{2}$ & $M_{2}$ & $M_{2}$ & $M_{2}$ & $M_{2}$ \\
Tool & $T_{5}$ & $T_{5}$ & $T_{5}$ & $T_{5}$ & $T_{5}$ & $T_{5}$ & $T_{5}$ & $T_{1}$ & $T_{1}$ & $T_{1}$ & $T_{1}$ & $T_{1}$ & $T_{8}$ & $T_{2}$ \\
TAD & $+Y$ & $+Y$ & $+Y$ & $-Y$ & $-Y$ & $-Y$ & $+X$ & $-Y$ & $-Z$ & $-Z$ & $+Z$ & $+Z$ & $-X$ & $-Z$ \\
\hline
\end{tabular}

Get the value of parameters by calculation as follows: the value of $T M C$ is 490 , the value of $T T C$ is 98 , the value of $M C C$ is 0 , the value of $N M C$ is 0 , the value of $T M C C$ is 0 , the value of $T C C$ is 120 , the value of $N T C$ is 3 , the value of $T T C C$ is 360 , the value of $N S$ is 9 , the value of $S C C$ is 15 , the value of $T S C C$ is 135 , the value of $T W C$ is 1083 , and the penalty cost is 200 .

Table 10. One of the best flexible process planning under condition (b) for the example part.

\begin{tabular}{cccccccccccccccc}
\hline No. & $O_{17}$ & $O_{18}$ & $O_{15}$ & $O_{9}$ & $O_{21}$ & $O_{8}$ & $O_{7}$ & $O_{1}$ & $O_{20}$ & $O_{16}$ & $O_{14}$ & $O_{23}$ & $O_{11}$ & $O_{5}$ \\
Feature & $F_{10}$ & $F_{11}$ & $F_{8}$ & $F_{5}$ & $F_{13}$ & $F_{4}$ & $F_{3}$ & $F_{1}$ & $F_{12}$ & $F_{9}$ & $F_{7}$ & $F_{14}$ & $F_{6}$ & $F_{2}$ \\
A-Op. & $A O_{101}$ & $A O_{111}$ & $A O_{81}$ & $A O_{51}$ & $A O_{131}$ & $A O_{41}$ & $A O_{31}$ & $A O_{1}$ & $A O_{122}$ & $A O_{91}$ & $A O_{72}$ & $A O_{141}$ & $A O_{61}$ & $A O_{23}$ \\
Machine & $M_{2}$ & $M_{2}$ & $M_{2}$ & $M_{2}$ & $M_{2}$ & $M_{2}$ & $M_{2}$ & $M_{2}$ & $M_{2}$ & $M_{2}$ & $M_{2}$ & $M_{2}$ & $M_{2}$ & $M_{2}$ \\
Tool & $T_{5}$ & $T_{5}$ & $T_{5}$ & $T_{5}$ & $T_{5}$ & $T_{5}$ & $T_{5}$ & $T_{1}$ & $T_{1}$ & $T_{1}$ & $T_{1}$ & $T_{1}$ & $T_{2}$ & $T_{8}$ \\
TAD & $-Y$ & $-Y$ & $+X$ & $+Y$ & $-X$ & $+Y$ & $+Y$ & $+Z$ & $-Z$ & $-Z$ & $-Z$ & $-Y$ & $-Z$ & $-Y$ \\
\hline
\end{tabular}

Get the value of parameters by calculation as follows: the value of $T M C$ is 490 , the value of $T T C$ is 98 , the value of $T C C$ is 120 , the value of $N T C$ is 3 , the value of $T T C C$ is 360 , the value of $T W C$ is 948 , and the penalty cost is 200 .

According to the above calculation example, the cost of machines changing once is higher than the cost of machining a feature, and the cost of tools changing once is higher than cost of machining a feature. Consequently, the solved optimal solution changes machine tools and cutting tools as little as possible under the premise of meeting the processing precedence constraints. The following two conditions are set in order to further test effectiveness of the algorithm: (c) all the machine tools and cutting tools are available, but the drilling operation must be finished by machine tool $M_{1}$; the value of $w_{1} \sim w_{5}$ in objective function is set to 1 . (d) all the machine tools and cutting tools are available, but the drilling operation must be finished by machine tool $M_{1}$; the values of parameters in objective function (17) are as follows: $w_{2}=w_{5}=0, w_{1}=w_{3}=w_{4}=1$. The parameter settings of algorithm are described above; the number of iterations $N_{c_{\max }}=100$, similarly running 10 times. The optimum value of every generation is shown in figures 8 and 9 . The optimum solution under the conditions of (c) and (d) is, respectively, shown in table 11 and table 12 . 


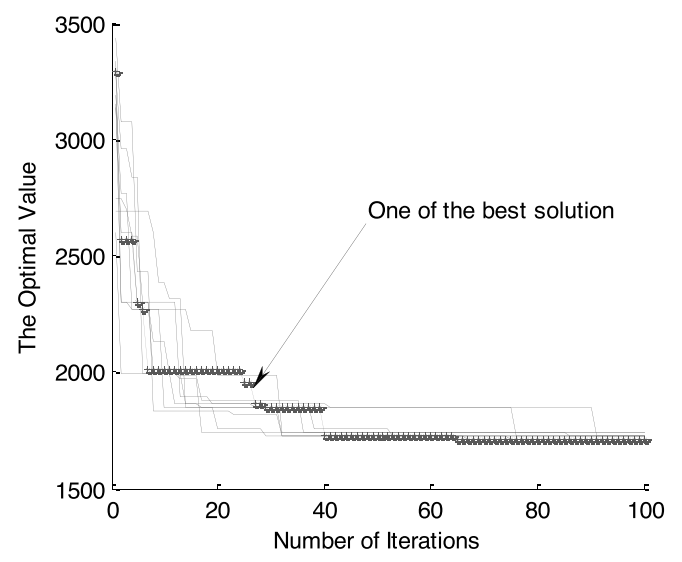

Fig. 8. Optimal value of each iteration in condition (c).

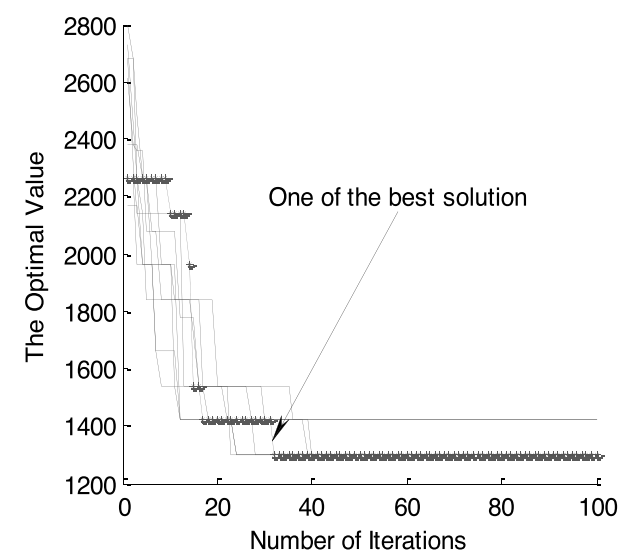

Fig. 9. Optimal value of each iteration in condition (d).

Table 10. One of the best flexible process planning under condition (c) for the example part.

$\begin{array}{llllllllllllllll}\text { No. } & O_{17} & O_{18} & O_{22} & O_{8} & O_{7} & O_{10} & O_{15} & O_{11} & O_{16} & O_{14} & O_{20} & O_{23} & O_{2} & O_{6}\end{array}$

Feature $\quad F_{10} \quad F_{11} \quad F_{13} \quad F_{4} \quad F_{3} \quad F_{5} \quad F_{8} \quad F_{6} \quad F_{9} \quad F_{7} \quad F_{12} \quad F_{14} \quad F_{1} \quad F_{6}$ A-Op. $\quad A O_{101} \quad A O_{111} \quad A O_{131} \quad A O_{41} \quad A O_{31} \quad A O_{51} \quad A O_{81} \quad A O_{61} \quad A O_{91} \quad A O_{72} \quad A O_{122} \quad A O_{141} \quad A O_{12} \quad A O_{62}$

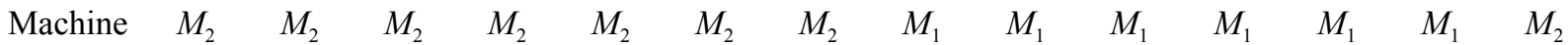
$\begin{array}{ccccccccccccccc}\text { Tool } & T_{5} & T_{5} & T_{5} & T_{5} & T_{5} & T_{5} & T_{5} & T_{2} & T_{1} & T_{1} & T_{1} & T_{1} & T_{1} & T_{8}\end{array}$ $\begin{array}{llllllllllllllll}\mathrm{TAD} & -Y & -Y & -Y & + & +Y & -Z & +X & +Z & -Z & -Z & -Z & -Y & -Z & -Z\end{array}$ 
Table 11. One of the best flexible process planning under condition (d) for the example part.

\begin{tabular}{cccccccccccccccc}
\hline No. & $O_{15}$ & $O_{17}$ & $O_{18}$ & $O_{22}$ & $O_{8}$ & $O_{9}$ & $O_{7}$ & $O_{12}$ & $O_{23}$ & $O_{13}$ & $O_{16}$ & $O_{19}$ & $O_{2}$ & $O_{4}$ \\
Feature & $F_{8}$ & $F_{10}$ & $F_{11}$ & $F_{13}$ & $F_{4}$ & $F_{5}$ & $F_{3}$ & $F_{6}$ & $F_{14}$ & $F_{7}$ & $F_{9}$ & $F_{12}$ & $F_{1}$ & $F_{2}$ \\
A-Op. & $A O_{81}$ & $A O_{101}$ & $A O_{111}$ & $A O_{132}$ & $A O_{41}$ & $A O_{51}$ & $A O_{31}$ & $A O_{62}$ & $A O_{141}$ & $A O_{71}$ & $A O_{91}$ & $A O_{121}$ & $A O_{12}$ & $A O_{22}$ \\
Machine & $M_{2}$ & $M_{2}$ & $M_{2}$ & $M_{2}$ & $M_{2}$ & $M_{2}$ & $M_{2}$ & $M_{1}$ & $M_{1}$ & $M_{1}$ & $M_{1}$ & $M_{1}$ & $M_{1}$ & $M_{2}$ \\
Tool & $T_{5}$ & $T_{5}$ & $T_{5}$ & $T_{5}$ & $T_{5}$ & $T_{5}$ & $T_{5}$ & $T_{2}$ & $T_{1}$ & $T_{1}$ & $T_{1}$ & $T_{1}$ & $T_{1}$ & $T_{8}$ \\
TAD & $+X$ & $-Y$ & $-Y$ & $-Y$ & $+Y$ & $+Y$ & $+Y$ & $-Z$ & $-Y$ & $+Z$ & $+Z$ & $-Z$ & $-Z$ & $+Y$ \\
\hline
\end{tabular}

Because the drilling operation can only be done by machine $M_{1}$, there are precedence constraints in the alternative machining operations; so, the machining tools need to be changed twice at least. The optimum value is 1928 (with the value of penalty cost being 200) under the condition of (c), and the optimum value is 1500 (with the value of penalty cost being 200) under the condition of (d). The results running ten times independently also show that it can search the minimum value, which can be searched currently many times.

\section{CONCLUSIONS}

An ant colony optimization method is proposed to solve the flexible process planning problem based on the dynamic updating of feasible alternative machining operations. The solution process is to search the route of the feasible alternative machining operations firstly and then select the processing operation resource on the basis of the obtained process route. The approach presented here has several advantages in the following aspects:

(1) In the description of flexible process planning, the alternative machining operations were generated by the TAD. The solving of the process planning is divided into two stages: the first stage is to determine the sequence of the alternative machining operations, and the second is to configure the manufacturing resources for the alternative machining operation sequences.

(2) In the processing of precedence constraint, the alternative machining operations were classified based on the precedence constraint matrix. As a result, it is very convenient to obtain the set of the feasible alternative machining operations, which was dynamically updated by setting the values of the row and the column corresponding to the last selected alternative machining operations to 0 in the precedence constraint matrix and the values of the corresponding diagonal were set to constant. Through the processing above, the next search space of ACO can be effectively limited.

(3) In the processing of the alternative machining operations, only one feasible alternative machining operation can be selected for the same machining process. By searching for the presence of a value of -1 in the row and column corresponding to the selected alternative machining operations in the precedence constraint matrix, the row and column of the corresponding feasible alternative machining operations are set to 0 and diagonals are set to constant, if -1 presents. As a result, only one feasible alternative machining operation can be selected in the same machining operation according to the definition of the feasible alternative machining operation.

(4) In terms of the performance of algorithms proposed, the results show that the proposed algorithm is superior to one performance index at least compared with the existing algorithms through specific examples. Moreover, the robustness of the algorithm is better.

In further study, the method of precedence constraint dynamic updating proposed in this paper can be incorporated 
with other intelligent algorithms to solve the problems of process planning and production scheduling, which could be compared with the existing algorithms to enhance the performance of the algorithm even more.

\section{ACKNOWLEDGMENT}

This work was supported by the National Natural Science Foundation of China (grant number 61403163) and the Zhejiang Provincial Natural Science Foundation of China (LQ14G010008, LY13E050021).

\section{REFERENCES}

Blanch, R., Pellicer, N. \& G. Romeu, M.L. 2011. Machining processes time calculating tool integrated in computer aided process planning (CAPP) for small and medium enterprises (SMEs). International Journal of Computer Integrated Manufacturing, 24(1):40-52.

Brown, K.N. \& Cagan, J. 1997. Optimized process planning by generative simulated annealing. Artificial Intelligence for Engineering Design Analysis \& Manufacturing, 11(3):219-235.

Ciurana, J., Garcia-Romeu, M.L., Ferrer, I. \& Casadesus, M. 2008. A model for integrating process planning and production planning and control in machining processes. Robotics and Computer-Integrated Manufacturing, 24(4):532-544.

Derek, Y. \& Debasish, D. 1996. A genetic algorithm application for sequencing operations in process planning for parallel machining. Iie Transactions, 28(1):55-68.

Dorigo, M., Maniezzo, V. \& Colomi, A. 1996. Ant system: Optimization by a colony of cooperating agents. IEEE Transactions on by system. Man and Cybemetics-part B 26(1):29-41.

Huang, W., Hu, Y. \& Cai, L. 2011. An effective hybrid graph and genetic algorithm approach to process planning optimization for prismatic parts. International Journal of Advanced Manufacturing Technology, 62(9-12):1219-1232.

Huang, F.L, Gu, J.M. \& Wang, H.Y. 2016. Simulation generation method of STEP-NC nonlinear process route based on manufacturing features. Computer Integrated Manufacturing Systems, 22(5):1245-1254.

Kiritsis, D. \& Porchet, M. 1996. A generic Petri net model for dynamic process planning and sequence optimization. Advances in Engineering Software, 25(1):61-71.

Krishna, A.G. \& Rao, K.M. 2006. Optimisation of operations sequence in CAPP using an ant colony algorithm. International Journal of Advanced Manufacturing Technology, 29(1-2):159-164.

Lee, D.H., Kiritsis, D. \& Xirouchakis, P. 2004. Iterative approach to operation selection and sequencing in process planning. International Journal of Production Research, 42(22):4745-4766.

Li, W.D., Ong, S.K. \& Nee, A.Y.C. 2002. Hybrid genetic algorithm and simulated annealing approach for the optimization of process plans for prismatic parts. International Journal of Production Research, 40(8):1899-1922.

Li, W.D., Ong, S.K.A. \& Nee, Y.C. 2004. Optimization of process plans using a constraint-based tabu search approach. International Journal of Production Research, 42(10):1955-1985.

Li, X.Y., Shao, X.Y. \& Gao, L. 2008. Optimization of flexible process planning by genetic programming. International Journal of Advanced Manufacturing Technology, 38(1):143-153.

Li, X., Gao, L. \& Wen, X. 2013. Application of an efficient modified particle swarm optimization algorithm for process planning. International Journal of Advanced Manufacturing Technology, 67(5-8):1355-1369.

Liu. X, Hong, Y., Ni, Z., Qi, J. \& Qiu, X. 2013. Process planning optimization of hole-making operations using ant colony algorithm. The International Journal of Advanced Manufacturing Technology, 69(1):753-769.

Liu, X.J., Yi, H. \& Ni, Z.H. 2013. Application of ant colony optimization algorithm in process planning optimization. Journal of Intelligent Manufacturing, 24(1):1-13.

Liu, X.J., Yi, H. \& Ni, Z.H. 2013. Application of ant colony optimization algorithm in process planning optimization[J]. Journal of Intelligent Manufacturing, 11(1):538-544.

Li, Y., Ba, L. \& Cao, Y. 2015. Research on integrated process planning and scheduling problem with consideration of multi- 
objectives. China Mechanical Engineering 26(17):2344-2351.

Lv, S. \& Qiao, L. 2013. A cross-entropy-based approach for the optimization of flexible process planning. International Journal of Advanced Manufacturing Technology, 68(9-12):2099-2110.

Ma, G.H., Zhang, Y.F. \& Nee, A.Y.C. 2000. A simulated annealing-based optimization algorithm for process planning. International Journal of Production Research, 38(12):2671-2687.

Musharavati, F. \& Hamouda, A.M.S. 2012. Simulated annealing with auxiliary knowledge for process planning optimization in reconfigurable manufacturing. Robotics and Computer-Integrated Manufacturing, 28(2):113-131.

Petrović, M., Mitić, M. \& Vuković, N. 2015. Chaotic particle swarm optimization algorithm for flexible process planning. International Journal of Advanced Manufacturing Technology, 154:1-21.

Qiao, L., Wang, X.Y. \& Wang, S.C. 2010. A GA-based approach to machining operation sequencing for prismatic parts. International Journal of Production Research, 38(14):3283-3303.

Seker, A., Erol, S. \& Botsali, R. 2013. A neuro-fuzzy model for a new hybrid integrated Process Planning and Scheduling system. Expert Systems with Applications, 40(40):5341-5351.

Sormaz, D.N. \& Khoshnevis, B. 2003. Generation of alternative process plans in integrated manufacturing systems. Journal of Intelligent Manufacturing, 14(6):509-526.

Usher, J.M. \& Fernandes, K.J. 1996. Dynamic process planning-The static phase. Journal of Materials Processing Technology, 61(1-2):53-58.

Wang,Y.F., Zhang, Y.F. \& Fuh, J.Y.H. 2011. A hybrid particle swarm based method for process planning optimisation. International Journal of Production Research, 50(1):277-292.

Wang, J.F., Wu, X. \& Fan, X. 2015. A two-stage ant colony optimization approach based on a directed graph for process planning. International Journal of Advanced Manufacturing Technology, 80(5-8):1-12.

Wen, X.Y., Li, X.Y. \& Gao, L. 2012. Honey bees mating optimization algorithm for process planning problem. Journal of Intelligent Manufacturing, 25(3):459-472.

Zhang, L. \& Wong, T.N. 2015. An object-coding genetic algorithm for integrated process planning and scheduling [J]. European Journal of Operational Research, 244(2):434-444.

Zoran, M. \& Milica, P. 2016. Application of modified multi-objective particle swarm optimisation algorithm for flexible process planning problem. International Journal of Computer Integrated Manufacturing, 1-21.

Submitted: $14 / 02 / 2017$

Revised: $\quad 17 / 06 / 2019$

Accepted: 19/06/2019 


\title{
نهج لتحسين مستعمرة النمل استناداً إلى مصفوفة قيود الأسبقية لتخطيط العمليات المرن
}

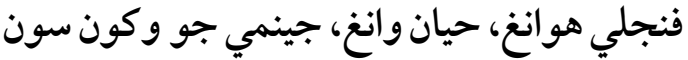 \\ كلية الهندسة ألميكانيكية والكهربائية، جامعة جياشينغ، جياشينغ، الصين
}

\section{الخلاصة}

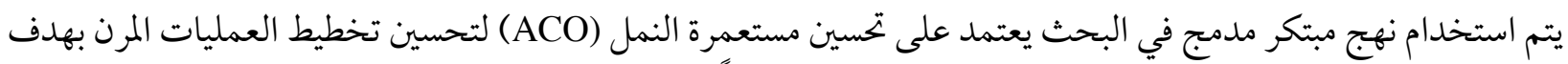

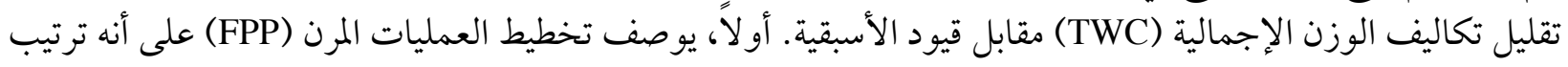

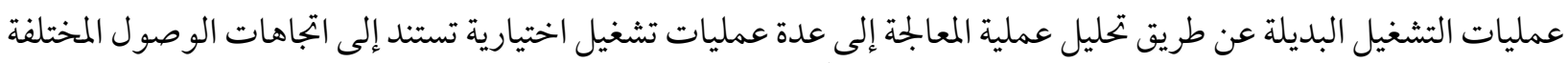

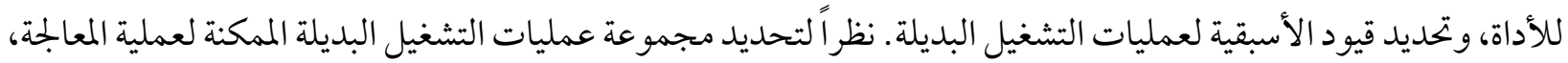

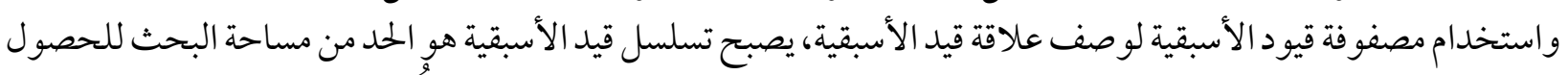

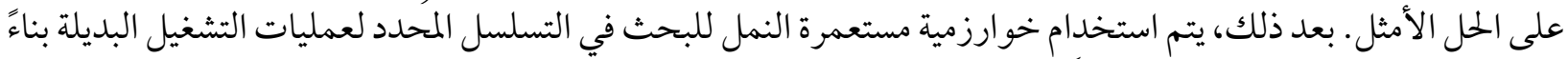

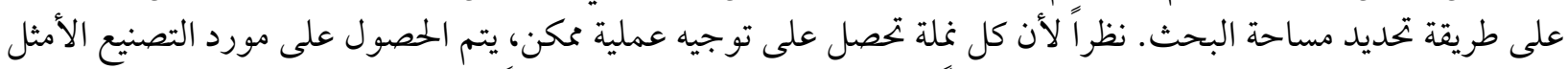

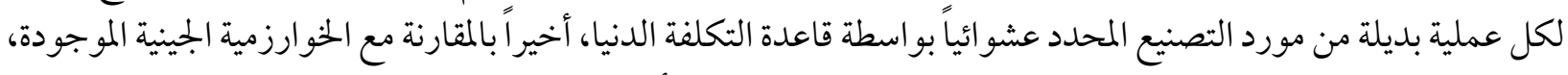

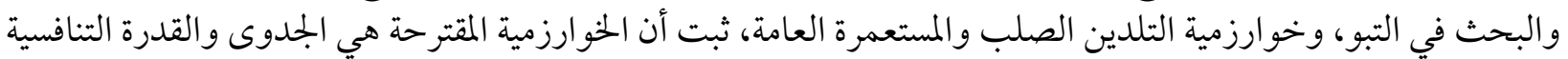

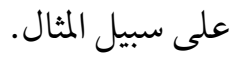

